\title{
A study of standing pressure waves within open and closed acoustic resonators.
}

\author{
C. Daniels, B. Steinetz, J. Finkbeiner, G. Raman, X. Li
}

The first section of the results presented herein was conducted on an axisymmetric resonator configured with open ventilation ports on either end of the resonator, but otherwise closed and free from obstruction. The remaining section presents the results of a similar resonator shape that was closed, but contained an axisymmetric blockage centrally located through the axis of the resonator. Ambient air was used as the working fluid. In each of the studies, the resonator was oscillated at the resonant frequency of the fluid contained within the cavity while the dynamic pressure, static pressure, and temperature of the fluid were recorded at both ends of the resonator. The baseline results showed a marked reduction in the amplitude of the dynamic pressure waveforms over previous studies due to the use of air instead of refrigerant as the working fluid. A sharp reduction in the amplitude of the acoustic pressure waves was expected and recorded when the configuration of the resonators was modified from closed to open. A change in the resonant frequency was recorded when blockages of differing geometries were used in the closed resonator, while acoustic pressure amplitudes varied little from baseline measurements. 


\title{
A Study of Standing Pressure Waves Within Open and Closed Acoustic Resonators.
}

\author{
C. Daniels \\ Ohio Aerospace Institute \\ Cleveland, OH 44142
}

\section{OAI}

B. Steinetz

NASA John H. Glenn Research Center

Cleveland, $\mathrm{OH} 44135$

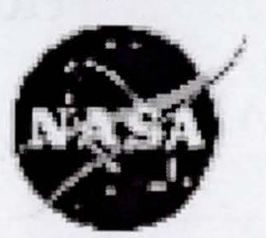

J. Finkbeiner, G. Raman, and X. Li Illinois Institute of Technology

Chicago, IL 60616

ILLINOIS INSTITUTE

OF TECHNOLOGY 


\section{Presentation Outline}

- Research Objective

- Background

- Baseline Configuration

$\rightarrow$ Experimental Setup

$\rightarrow$ Results

- Open Resonator Configuration

$\rightarrow$ Experimental Setup

$\rightarrow$ Results

- Closed Configuration with Blockages

$\rightarrow$ Experimental Setup

$\rightarrow$ Results

- Comparison of Results

- Conclusions 


\section{Research Objective}

- Extend the research of:

$\rightarrow$ Lawrenson, et al. (1998)

$\rightarrow$ Ilinskii, et al. (1998)

$\rightarrow$ Chun, et al. (2000)

- Determine if high-amplitude standing pressure waves can be generated:

$\rightarrow$ using air as the working fluid

$\rightarrow$ in resonators containing blockages

$\rightarrow$ in resonators containing ventilation holes

- Goal is to determine applicability to NASA's flow control systems 


\section{Background}

- Dr. Timothy Lucas discovered a method to produce high-amplitude acoustic waves in resonators.

- Previously published work focused mainly on using refrigerant as the working fluid.

- NASA's need for methods of flow control has driven the research towards applicability of using air as the working fluid. 


\section{Dimensionless Variables}

Dimensionless Pressure

$\rightarrow \mathbf{P} / \mathbf{P o}=\mathrm{P}_{\text {Instantaneous }} / \mathrm{P}_{\text {AVe Quiet condition }}$

$\rightarrow \mathbf{P}_{\text {MaX }} / \mathbf{P o}=\mathrm{P}_{\text {CYCle maXimum }} / \mathrm{P}_{\text {AVE Quiet Condition }}$

- Dimensionless Frequency

$\rightarrow \Omega=2 \cdot f \cdot 1_{\text {RESONATOR }} /\left(\gamma \cdot 8314 \cdot \mathrm{T}_{\mathrm{K}} / \mathrm{MW}\right)^{1 / 2}$

- Dimensionless Time

$\rightarrow \tau=\mathrm{f} \cdot \mathrm{t}$ 


\section{Baseline Configuration}




\section{Baseline Configuration: Experimental Setup}

- $\quad$ Electrodynamic Shaker Table

$\rightarrow \quad 500 \mathrm{lbf}(2220 \mathrm{~N})$ capacity

- Conical Resonator

$\rightarrow \quad \mathrm{r}(\mathrm{z})=0.0056+0.2680 \cdot \mathrm{z}[\mathrm{m}]$

$\rightarrow \quad$ Aluminum $7075 \mathrm{~T} 6$ with 0.14 inch $\left(3.6 \times 10^{-3} \mathrm{~m}\right)$ wall thickness

$\rightarrow \quad$ Containing air (ambient conditions)

- Instrumentation

Each end of the resonator contains:
A. Dynamic pressure sensors (2)
B. Static pressure transducers (2)
C. Accelerometer (2)
D. Thermocouples (2)

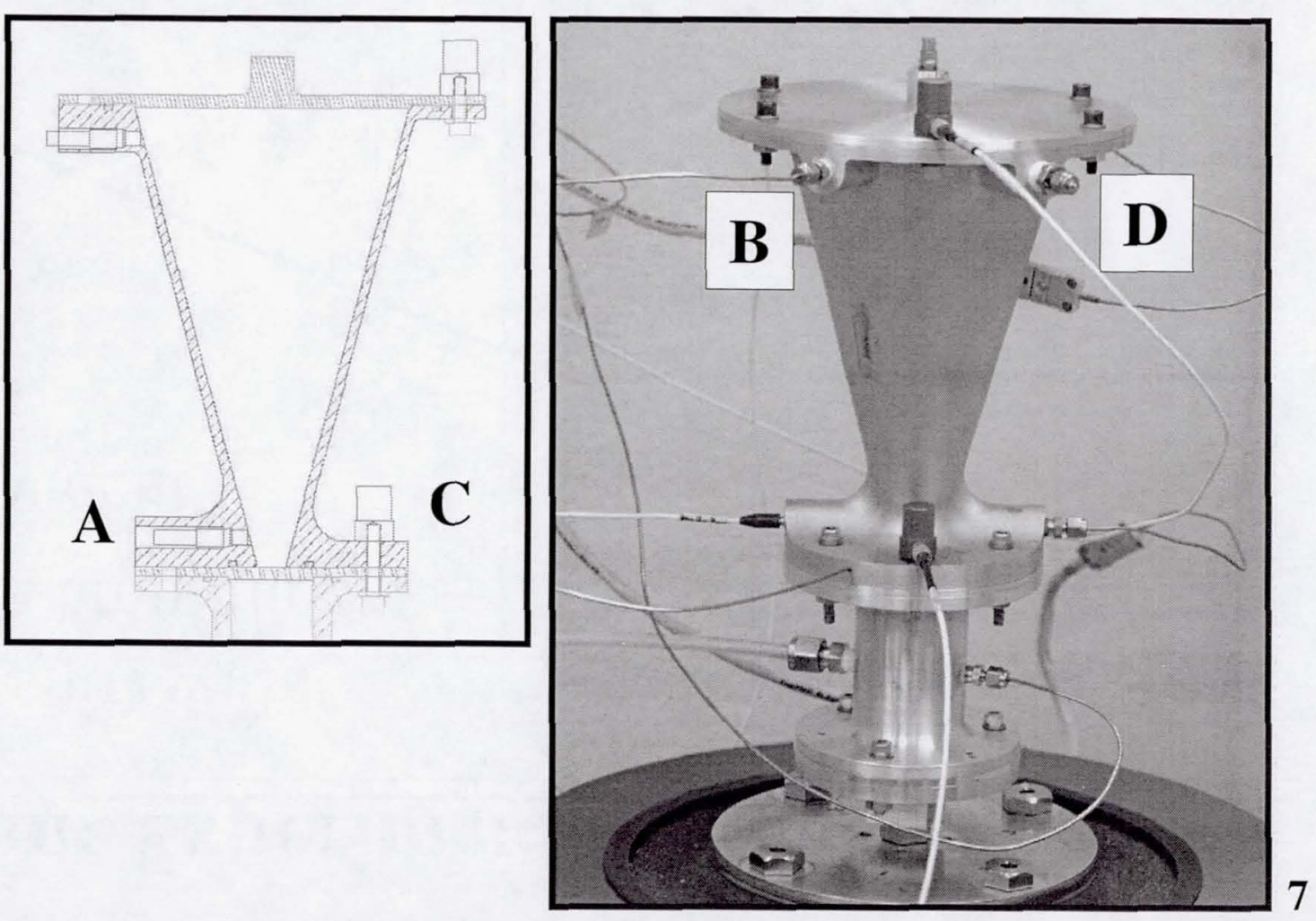




\section{Baseline Configuration: Experimental Results}

- Non-linear frequency shift with increasing acceleration amplitude

- Moderate hysteresis evident (hardening)

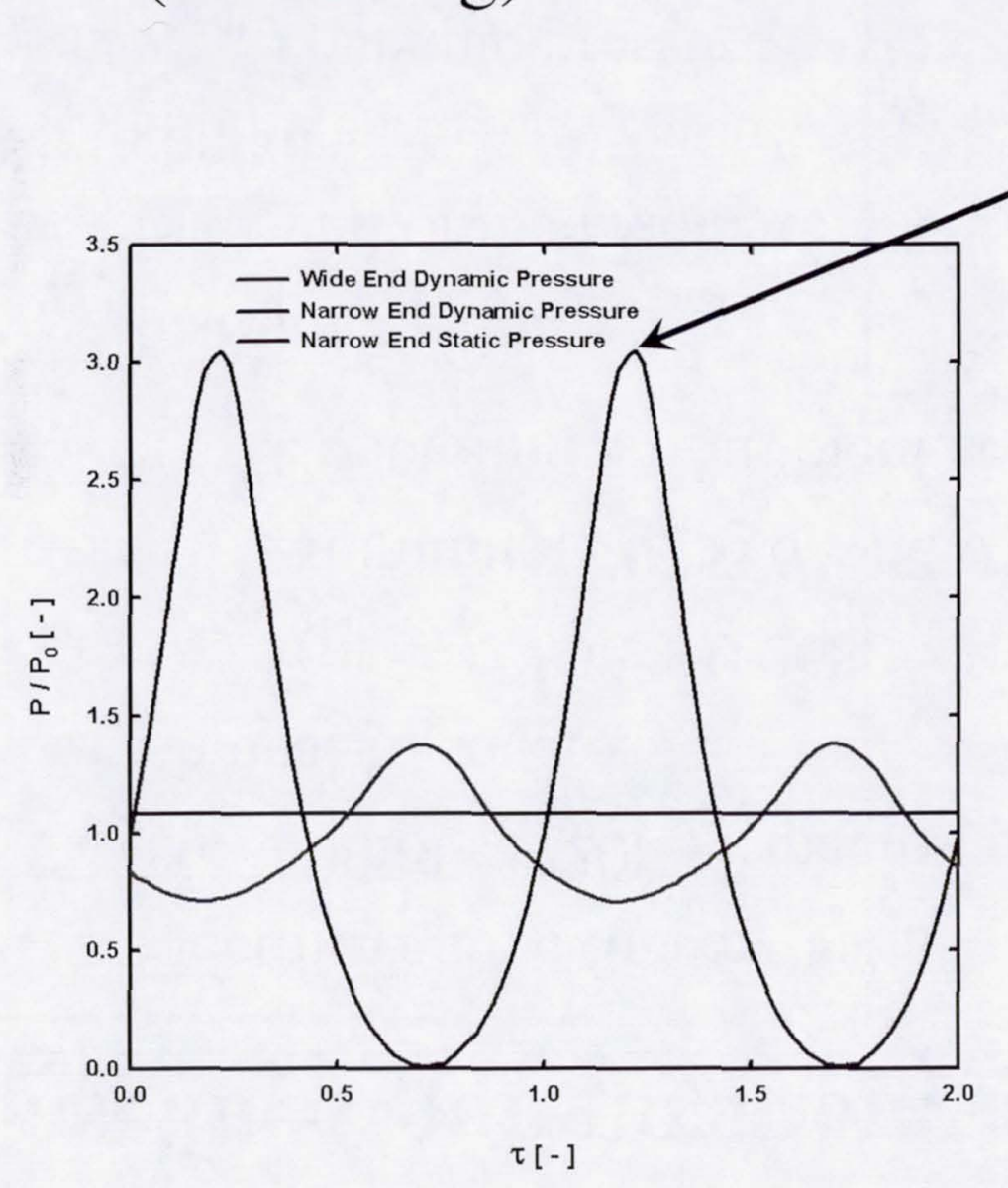

- Constant max acceleration: $80 \mathrm{~g}$

- $\mathrm{P}_{\mathrm{o}}=100.2 \mathrm{kPa}$

- No microshocks evident

- $\mathrm{P}_{\mathrm{MAX}} / \mathrm{P}_{0}=3.05 \quad(306 \mathrm{kPa})$

- Static Pressure rise of $\sim 6.5 \mathrm{kPa}$ 
Open Resonator Configuration 


\section{Open Resonator Configuration: Experimental Setup}

- Identical hardware and instrumentation used in baseline experiments

- End caps

$\rightarrow$ Aluminum 7075T6

$\rightarrow 0.188$ inch $\left(4.77 \times 10^{-3} \mathrm{~m}\right)$ thickness

- Additionally

$\rightarrow$ Wide end cap: one (1) ventilation hole $\phi 0.100$ in $\left(\phi 2.54 \times 10^{-3} \mathrm{~m}\right)$

$\rightarrow$ Narrow end cap: eight (8) ventilation holes $\phi 0.025$ in $\left(\phi 6.35 \times 10^{-4} \mathrm{~m}\right)$

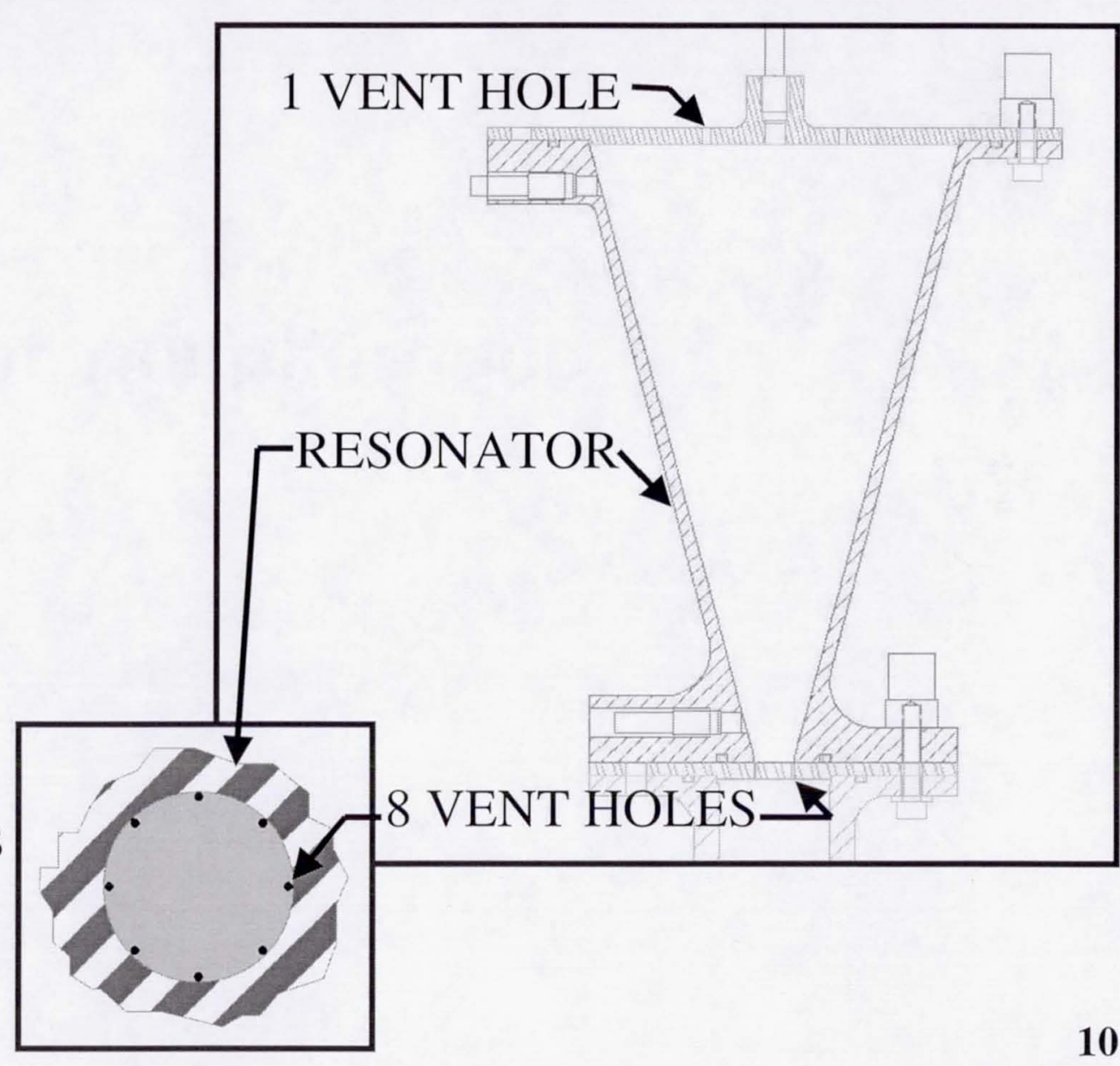




\section{Open Configuration: Experimental Results}

- Max acceleration: $80 \mathrm{~g}$

- No apparent hysteresis

- $\mathrm{P}_{\text {MAX }} / \mathrm{P}_{0}=1.48(148 \mathrm{kPa})$

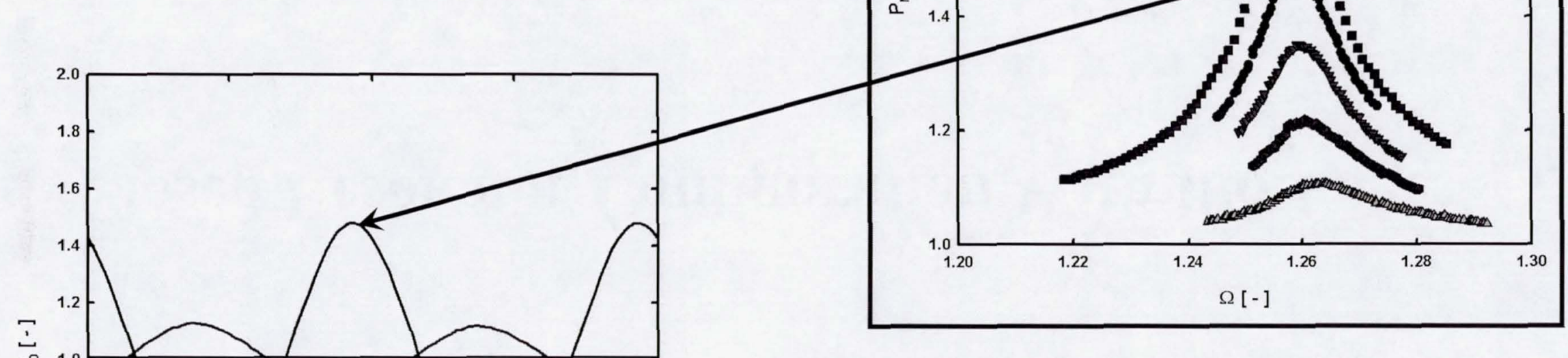

- No microshocks evident

- $\mathrm{P}_{\mathrm{o}}=99.2 \mathrm{kPa}$

- Static Pressure rise of $\sim 0.8 \mathrm{kPa}$ 


\section{Closed Resonator Configuration With Blockages}




\section{Closed Configuration w/ Blockages: Experimental Setup}

- Identical hardware and instrumentation used in Baseline experiments

- Baseline end caps (no ventilation holes)

- Additionally

$\rightarrow$ Centrally located cylindrical blockage

- $\phi 0.443$ inch $(1.356 \mathrm{~cm})$

- $\phi 0.433$ inch $(1.331 \mathrm{~cm})$

- $\phi 0.423$ inch $(1.306 \mathrm{~cm})$

- $\phi 0.403$ inch $(1.255 \mathrm{~cm})$

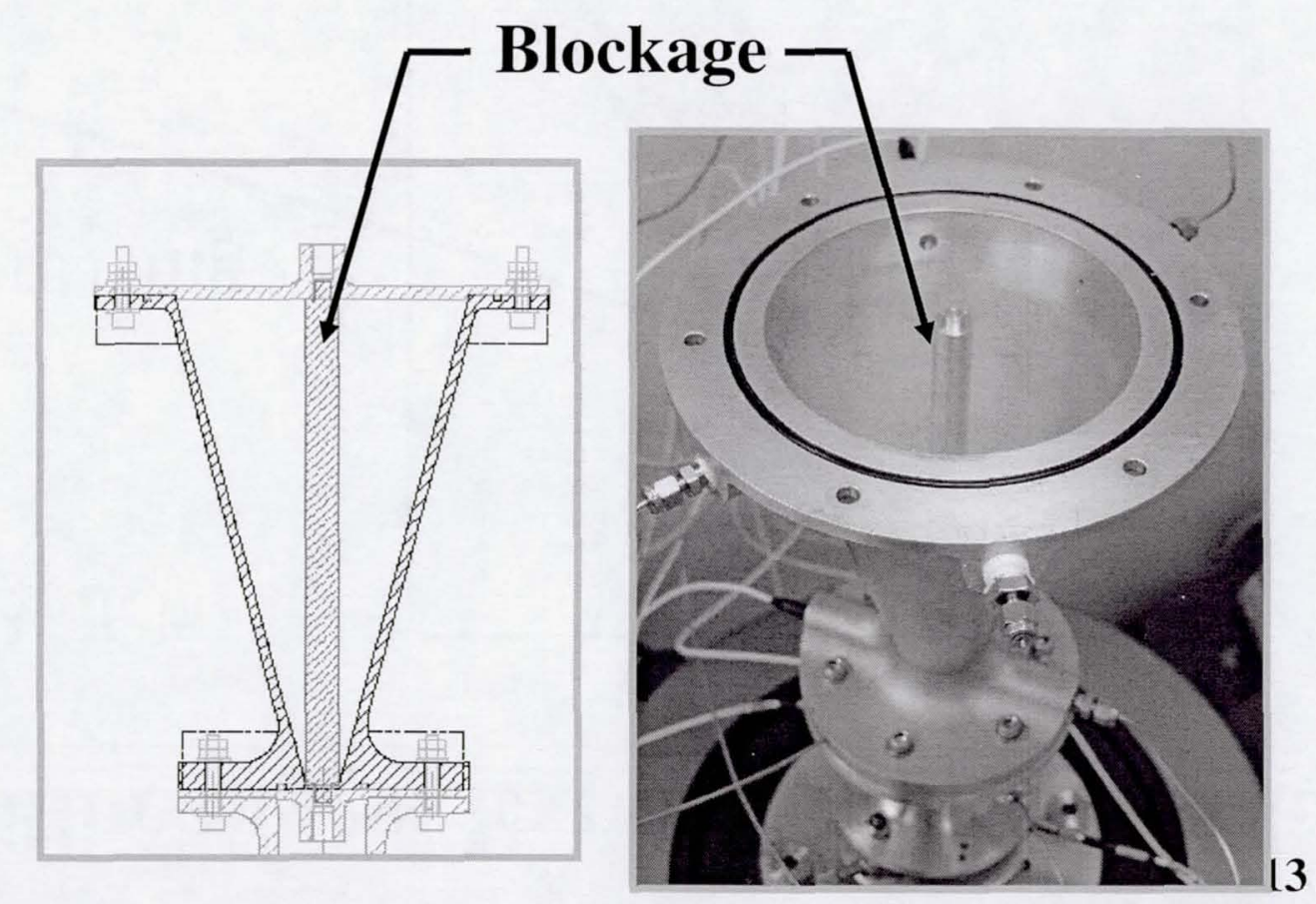




\section{Closed Configuration w/Blockages: Experimental Results}

- Blockage Diameter: $\phi 0.403$ inch $(1.255 \mathrm{~cm})$

- No apparent hysteresis

- No frequency shift with increasing acceleration amplitude
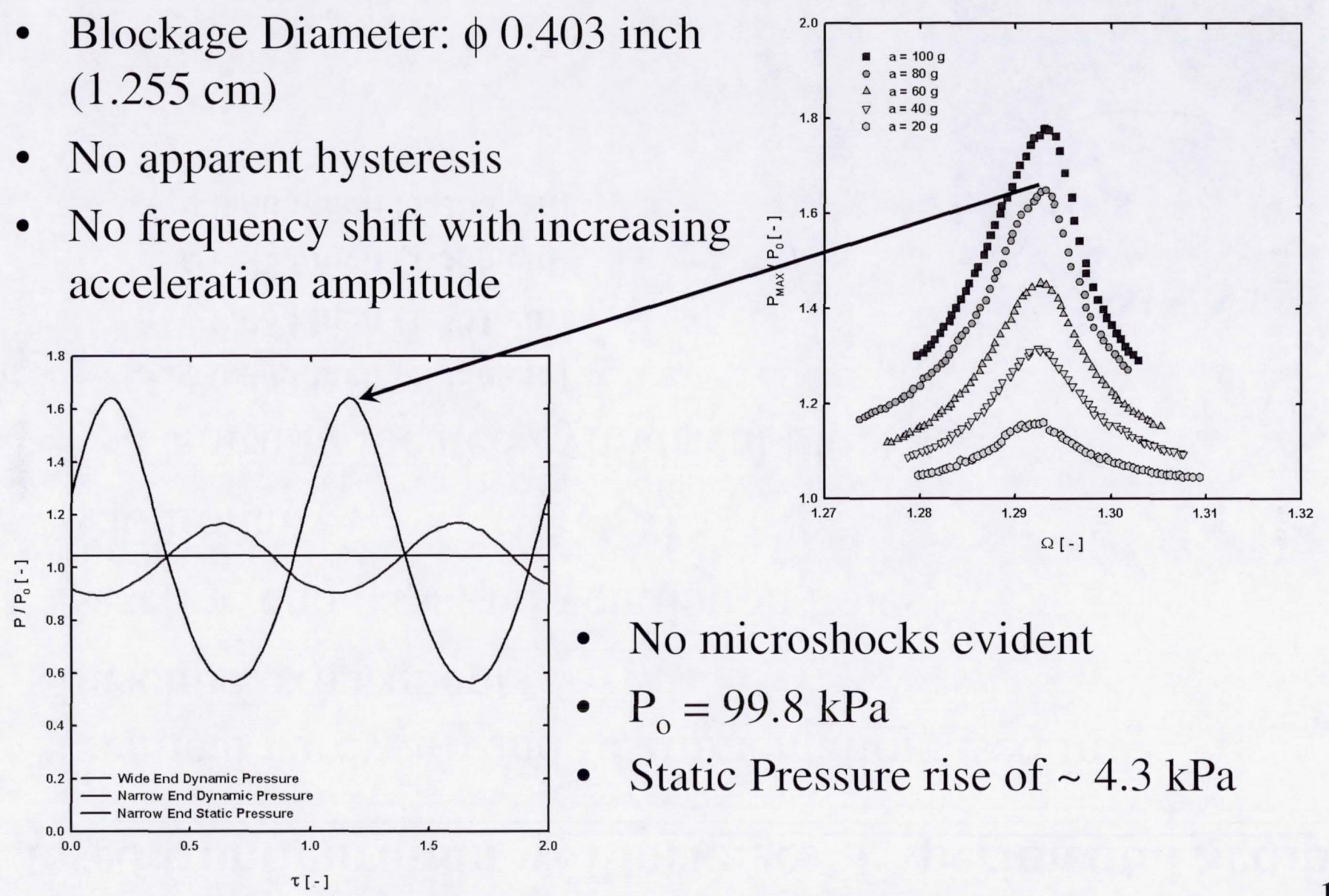

- No microshocks evident

- $\mathrm{P}_{\mathrm{o}}=99.8 \mathrm{kPa}$

- Static Pressure rise of $\sim 4.3 \mathrm{kPa}$ 


\section{Closed Configuration w/Blockages: Experimental Results}

- Constant maximum sinusoidal acceleration: $80 \mathrm{~g}$

- Increasing blockage diameter:

$\rightarrow$ Reduces $\mathrm{P}_{\mathrm{MAX}}$

- $P_{\text {MAX }} / P_{0}=1.65(\phi 0.403$ inch $)$

- $P_{\text {MAX }} / P_{0}=1.57(\phi 0.443$ inch $)$

$\rightarrow$ Increases fundamental resonant frequency

- $\Omega_{1}=1.293(\phi 0.403$ inch $)$

- $\Omega_{1}=1.299(\phi 0.443$ inch $)$

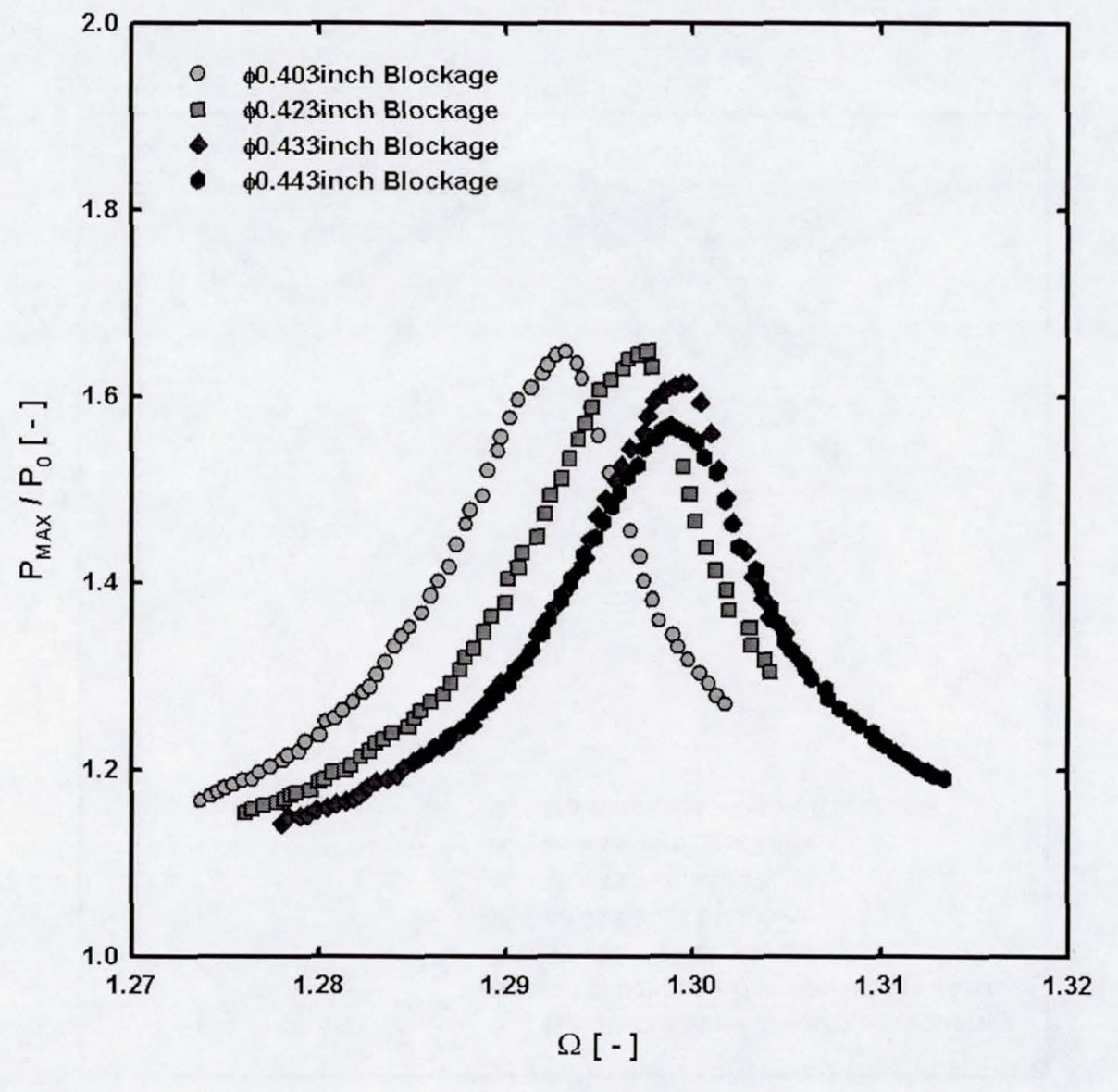




\section{Comparison of Results}

- Maximum

Acceleration

Amplitude: $80 \mathrm{~g}$

- From the baseline configuration:

$\rightarrow \mathrm{P}_{\text {MAX }}$ reduced $49 \%$ with addition of openings

$\rightarrow \mathrm{P}_{\text {MAX }}$ reduced 54\% with addition of blockages

$\rightarrow \Omega$ increased $2 \%$ with addition of

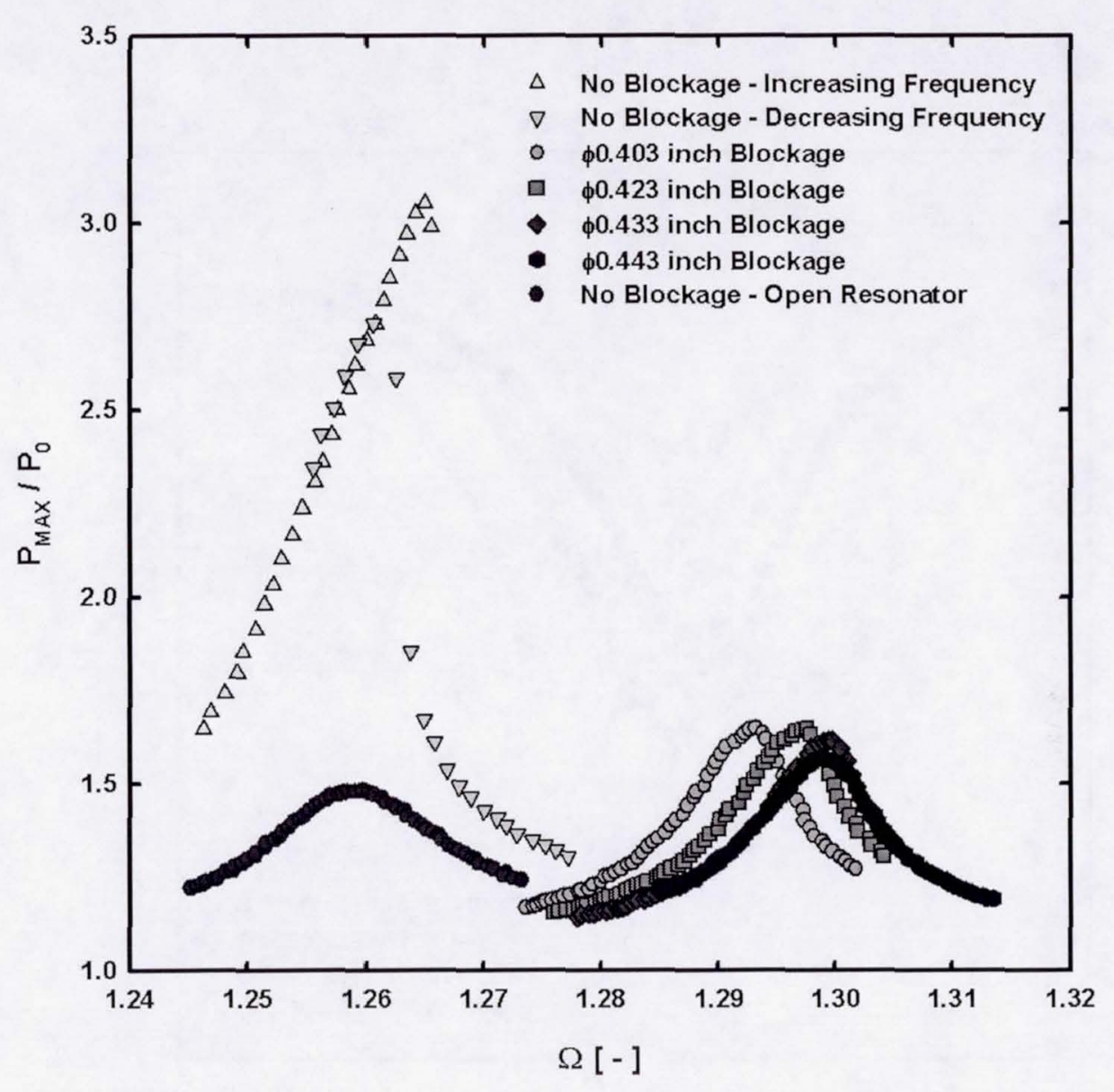
blockages 


\section{End of Presentation}




\section{Conclusions}

1. Standing waves with maximum pressures of $306 \mathrm{kPa}$ have been produced in resonators containing ambient pressure air.

2. While the addition of holes to the resonator does reduce the magnitude of the acoustic waves produced, their addition does not prohibit the generation of large magnitude standing waves.

3. The addition of structures inside the resonator shifts the fundamental frequency and decreases the amplitude of the generated pressure waves. 